\title{
THE GEODESIC RAY TRANSFORM ON RIEMANNIAN SURFACES WITH CONJUGATE POINTS
}

\author{
FRANÇOIS MONARD, PLAMEN STEFANOV, AND GUNTHER UHLMANN
}

\begin{abstract}
We study the geodesic X-ray transform $X$ on compact Riemannian surfaces with conjugate points. Regardless of the type of the conjugate points, we show that we cannot recover the singularities and therefore, this transform is always unstable (ill-posed). We describe the microlocal kernel of $X$ and relate it to the conjugate locus. We present numerical examples illustrating the cancellation of singularities. We also show that the attenuated X-ray transform is well posed if the attenuation is positive and there are no more than two conjugate points along each geodesic; but still ill-posed, if there are three or more conjugate points. Those results follow from our analysis of the weighted X-ray transform.
\end{abstract}

\section{INTRODUCTION}

The purpose of this paper is to study the X-ray transform on Riemannian surfaces over geodesics with conjugate points. Let $\gamma_{0}$ be a fixed directed geodesic on a Riemannian manifold of dimension $n \geq 2$, and let $f$ be a function which support does not contain the endpoints of $\gamma_{0}$. We study first the following local problem: what part of the wave front set $\mathrm{WF}(f)$ of $f$ can be obtained from knowing the wave front of the (possibly weighted) integrals

$$
X f(\gamma)=\int \kappa(\gamma(s), \dot{\gamma}(s)) f(\gamma(s)) \mathrm{d} s
$$

of $f$ along all (directed) geodesics $\gamma$ close enough to $\gamma_{0}$ ? The analysis can be easily generalized to more general geodesic-like curves as in [10] or to the even more general case of "regular exponential maps" [40] as in [37]. For the simplicity of the exposition, we consider the geodesic case only. Since $X$ has a Schwartz kernel with singularities of conormal type, $X f$ could only provide information about $\mathrm{WF}(f)$ near the conormal bundle $N^{*} \gamma_{0}$ of $\gamma_{0}$. It is well known that if there are no conjugate points on $\gamma_{0}$, we can in fact recover $\mathrm{WF}(f)$ near $N^{*} \gamma_{0}$. This goes back to Guillemin [14, 15] for integral transforms satisfying the Bolker condition, see also [26]. The latter allows the use of the clean intersection calculus of Duistermaat and Guillemin [9] to show that $X^{*} X$ is a pseudodifferential operator $(\Psi D O)$, elliptic when $\kappa \neq 0$. In the geodesic case under consideration, a microlocal study of $X f$ has been done in [31, 32, 10, 35, 37, 39], and in some of those works, $f$ can even be a tensor field.

In the presence of conjugate points, the Bolker condition fails, see also Proposition 3.1. Then $X^{*} X$ is not a $\Psi D O$ anymore, and the standard arguments do not apply. Moreover, one can easily construct examples with $\kappa=1$ based on the sphere [37] where the localized X-ray transform has an infinitely dimensional kernel of distributions singular at or near $N^{*} \gamma_{0}$. Two delta functions of opposite signs placed at two anti-podal points is one such case. In [37], the second and the

Date: May 4, 2014.

First author partly supported NSF grant No. 1265958.

Second author partly supported by a NSF Grant DMS-1301646.

Third author partly supported NSF grant No. 1265958 and a Simons Fellowship. 
third author studied this question assuming that the conjugate points on $\gamma_{0}$ are of fold type, i.e., $v \mapsto \exp _{x} v$ has fold type singularities $[1,12]$. In dimension $n=2$, we proved there that there is a loss of $1 / 4$ derivatives, and we describes the microlocal kernel of $X$ modulo that loss. The analysis there was based on the specific properties of the fold conjugate points, and was focused on understanding the structure of $X^{*} X$ as a sum of a $\Psi D O$ plus a Fourier Integral Operator (FIO) associated with the conormal bundle $N^{*} \Sigma$ of the conjugate locus $\Sigma$, see next section. The microlocal structure of $X^{*} X$ in all dimensions was also studied by Sean Holman [17]. In this work, we show that there is a loss of all derivatives, and that this is true for all possible types of conjugate points. Instead of studying $X^{*} X$, we study $X$ itself as an FIO; show that the singularities in the kernel are related by a certain FIO; and describe its canonical relation as a generalization of $N^{*} \Sigma^{\prime}$ even if the latter may not be smooth.

Since the answer to the local problem in two dimensions is affirmative when there are no conjugate points, and we show that it is negative when there are, the present paper gives a complete answer to the local problem for $n=2\left(X f\right.$ known near a single $\left.\gamma_{0}\right)$ with the exception of the borderline case when the conjugate points are on $\partial M$.

The global problem, recovery of $\mathrm{WF}(f)$, and ultimately $f$ from $X f$ known for all (or for a "large" set of) geodesics is different however. Let $M$ be two dimensional and non-trapping so that $X f$ is defined globally. The union of $N^{*} \gamma$ for all directed geodesics in $M$ is a double cover of $T^{*} M \backslash 0$, and for each $(x, \xi) \in T^{*} M \backslash 0, \mathrm{WF}(f)$ there can be possibly resolved by $X f$ known near each of the two directed geodesics through $x$ normal to $\xi$. We therefore have a system of two equations, and then that system is solvable for a pair of conjugate points, provided that some determinant does not vanish, see (5.1). If the weight is even, for example, or more generally if the matrix in (5.1) has rank one, then the global problem is equivalent to the local one. Condition (5.1) is naturally satisfied for the geodesic attenuated X-ray transform with positive attenuation. Those two equations however are not enough to resolve the singularities at three points conjugate to each other, and then we still cannot recover $\mathrm{WF}(f)$. Those results are formulated in Section 5.

Recovery of $\mathrm{WF}(f)$ is directly related to the question of stability of the geodesic X-ray transform (or any other linear map) - given $X f$, can we recover $f$ in a stable way? We do not study the uniqueness question here but we will just mention that if $(M, g)$ is real analytic, in many cases, even ones with conjugate points, one can have injectivity based on analytic microlocal arguments. An example of that is a small tubular neighborhood of $\gamma_{0}$ as above; then the geodesics normal or close to normal to $\gamma_{0}$ carry enough information to prove injectivity based on analyticity arguments as in $[10,35,5,21]$, for example. Stability however, will be always lost for even weights, for example, if there are conjugate points. The attenuated X-ray transform is stable with one or no conjugate point along each geodesic, and unstable otherwise. The term stable still makes sense even if there is no injectivity; then it indicates that estimate (4.7) holds.

This linear problem is also in the heart of the non-linear problem of recovery a metric or a sound speed (a conformal factor) from the lengths of the geodesics measured at the boundary (boundary rigidity) or from knowledge of the lens relation (lens rigidity), see, e.g., [7, 6, 8, 25, 28, 36, 34, 32, 29]; or from knowledge of the hyperbolic Dirichlet-to-Neumann (DN) map [3, 4, 23, 27, 33, 30, 2]. It is the linearization of the first two ( $f$ is a tensor field then); and the lens relation is directly related to the DN map and its canonical relation as an FIO. Although fully non-linear methods for uniqueness (up to isometry) exist, see, e.g., [3], stability is always derived from stability of the linearization. Very often, see for example [32], even uniqueness is derived from injectivity and stability of the linearization, see also [30] for an abstract treatment. Understanding the stability of $X$ is therefore fundamental for all those problems. Recovery of lower order terms like a magnetic and an electric 
fields from the associated DN maps leads to X-ray transforms of those fields along the geodesics [23] as well.

In seismology, recovery of the jumps between layers, which mathematically are conormal singularities, is actually the main goal. The model then is a linearized map like $X$ or a linearized DN map; and the goal is to recover the visible part of the wave front set.

In dimensions $n \geq 3$, the problem is over-determined. If there is a complete set of geodesics without conjugate points which conormal bundle covers $T^{*} M$, then $\mathrm{WF}(f)$ can be recovered [35]. In case this is not true or when we have local information only, there can be instability, for example for metrics of product type. In [37], we formulated a condition for fold conjugate points, under which singularities can still be recovered because then the "artifact" (the $F_{21}$ term in Theorem 4.3) are of lower order. At present it is not clear however if there are metrics satisfying that condition; and even if there are, the analysis does not cover non-fold conjugate points. Therefore, this problem remains largely open in dimensions $n \geq 3$.

The structure of the paper is as follows. In Section 2, we present some facts about the structure of the conjugate points. In Section 3, we characterize $X$ as an FIO. The main theoretical results are in Section 4 and Section 5. Numerical evidence is presented in Section 6.

\section{Regular EXPONENTial MAPS AND THEIR GENERIC SINGUlARIties}

2.1. Regular exponential maps. Let $(M, g)$ be a fixed $n$-dimensional Riemannian manifold. We recall some known facts about the structure of the conjugate points, mostly due Warner [40]. Most of the terminology comes from there but some is taken from [37].

2.2. Generic properties of the conjugate locus. We recall here the main result by Warner [40] about the regular points of the conjugate locus of a fixed point $p$. In fact, Warner considers more general exponential type of maps but we restrict our attention to the geodesic case (see also [37] for the general case). The tangent conjugate locus $S(p)$ of $p$ is the set of all vectors $v \in T_{p} M$ so that $\mathrm{d}_{v} \exp _{p}(v)$ (the differential of $\exp _{p}(v)$ w.r.t. $v$ ) is not an isomorphism. We call such vectors conjugate vectors at $p$ (called conjugate points in [40]). The kernel of $\mathrm{d}_{v} \exp _{p}(v)$ is denoted by $N_{p}(v)$. It is part of $T_{v} T_{p} M$ that we identify with $T_{p} M$. By the Gauss lemma, $N_{p}(v)$ is orthogonal

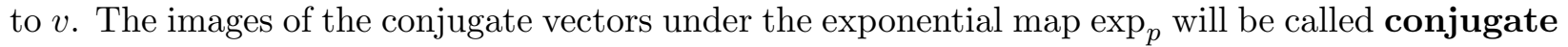
points to $p$. The image of $S(p)$ under the exponential map $\exp _{p}$ will be denoted by $\Sigma(p)$ and called the conjugate locus of $p$, which may not be smooth everywhere. Note that $S(p) \subset T_{p} M$, while $\Sigma(p) \subset M$. We always work with $p$ near a fixed $p_{0}$ and with $v$ near a fixed $v_{0}$. Set $q_{0}=\exp _{p_{0}}\left(v_{0}\right)$. Then we are interested in $S(p)$ restricted to a small neighborhood of $v_{0}$, and in $\Sigma(p)$ near $q_{0}$. Note that $\Sigma(p)$ may not contain all points near $q_{0}$ conjugate to $p$ along some geodesic; and may not contain even all of those along $\exp _{p_{0}}\left(t v_{0}\right)$ if the later self-intersects - it contains only those that are of the form $\exp _{p}(v)$ with $v$ close enough to $v_{0}$.

We denote by $\Sigma$ the set of all conjugate pairs $(p, q)$ localized as above. In other words, $\Sigma=$ $\{(p, q) ; q \in \Sigma(p)\}$, where $p$ runs over a small neighborhood of $p_{0}$. Also, we denote by $S$ the set $(p, v)$, where $v \in S(p)$.

A regular conjugate vector $v$ at $p$ is defined by the requirement that there exists a neighborhood $U$ of $v$, so that any radial ray $\{t v\}$ of $T_{p} M$ contains at most one conjugate vector in $U$. The regular conjugate locus then is an everywhere dense open subset of the conjugate locus and is an embedded $(n-1)$-dimensional manifold. The order of a conjugate vector as a singularity of $\exp _{p}$ (the dimension of the kernel of the differential) is called an order of the conjugate vector.

In [40, Thm 3.3], Warner characterized the conjugate vectors at a fixed $p_{0}$ of order at least 2 , and some of those of order 1 , as described below. Note that in $\left(B_{1}\right)$, one needs to postulate that $N_{p_{0}}(v)$ 
remains tangent to $S\left(p_{0}\right)$ at points $v$ close to $v_{0}$ as the latter is not guaranteed by just assuming that it holds at $v_{0}$ only.

(F) Fold conjugate vectors. Let $v_{0}$ be a regular conjugate vector at $p_{0}$, and let $N_{p_{0}}\left(v_{0}\right)$ be one-dimensional and transversal to $S\left(p_{0}\right)$. Such singularities are known as fold singularities. Then one can find local coordinates $\xi$ near $v_{0}$ and $y$ near $q_{0}$ so that in those coordinates, $y=\exp _{p_{0}} \xi$ is given by

$$
y^{\prime}=\xi^{\prime}, \quad y^{n}=\left(\xi^{n}\right)^{2} .
$$

Then $\operatorname{det} \mathrm{d}_{\xi} \exp _{p_{0}} \xi=2 \xi^{n}$ and

$$
S\left(p_{0}\right)=\left\{\xi^{n}=0\right\}, \quad N_{p_{0}}\left(v_{0}\right)=\operatorname{span}\left\{\partial / \partial \xi^{n}\right\}, \quad \Sigma\left(p_{0}\right)=\left\{y^{n}=0\right\} .
$$

Since the fold condition is stable under small $C^{\infty}$ perturbations, as follows directly from the definition, those properties are preserved under a small perturbation of $p_{0}$.

$\left(\mathbf{B}_{1}\right)$ Blowdown of order 1 . Let $v_{0}$ be a regular conjugate vector at $p_{0}$ and let $N_{p_{0}}(v)$ be one-dimensional. Assume also that $N_{p_{0}}(v)$ is tangent to $S\left(p_{0}\right)$ for all regular conjugate $v$ near $v_{0}$. We call such singularities blowdown of order 1 . Then locally, $y=\exp _{p_{0}} \xi$ is represented in suitable coordinates by

$$
y^{\prime}=\xi^{\prime}, \quad y^{n}=\xi^{1} \xi^{n}
$$

Then $\operatorname{det} \mathrm{d}_{\xi} \exp _{p_{0}} \xi=\xi^{1}$ and

$$
S\left(p_{0}\right)=\left\{\xi^{1}=0\right\}, \quad N_{p_{0}}\left(v_{0}\right)=\operatorname{span}\left\{\partial / \partial \xi^{n}\right\}, \quad \Sigma\left(p_{0}\right)=\left\{y^{1}=y^{n}=0\right\} .
$$

Even though we postulated that the tangency condition is stable under perturbations of $v_{0}$, it is not stable under a small perturbation of $p_{0}$, and the type of the singularity may change then. In some symmetric cases, one can check directly that the type is locally preserved.

$\left(\mathbf{B}_{k}\right)$ Blowdown of order $k \geq 2$. Those are regular conjugate vectors in the case where $N_{p_{0}}\left(v_{0}\right)$ is $k$-dimensional, with $2 \leq k \leq n-1$. Then in some coordinates, $y=\exp _{p_{0}} \xi$ is represented as

$$
\begin{array}{rlrl}
y^{i} & =\xi^{i}, & & i=1, \ldots, n-k \\
y^{i}=\xi^{1} \xi^{i}, & & i=n-k+1, \ldots, n .
\end{array}
$$

Then $\operatorname{det} d_{\xi} \exp _{p_{0}} \xi=\left(\xi^{1}\right)^{k}$ and

$$
\begin{aligned}
& S\left(p_{0}\right)=\left\{\xi^{1}=0\right\}, \quad N_{p_{0}}\left(v_{0}\right)=\operatorname{span}\left\{\partial / \partial \xi^{n-k+1}, \ldots, \partial / \partial \xi^{n}\right\}, \\
& \Sigma\left(p_{0}\right)=\left\{y^{1}=y^{n-k+1}=\cdots=y^{n}=0\right\} .
\end{aligned}
$$

In particular, $N_{p_{0}}\left(v_{0}\right)$ must be tangent to $S\left(p_{0}\right)$, see also [40, Thm 3.2]. This singularity is unstable under perturbations of $p_{0}$, as well. A typical example are the antipodal points on $S^{n}, n \geq 3$; then $k=n-1$. Note that in this case, the defining equation $\operatorname{det} \mathrm{d}_{v} \exp _{p_{0}} \xi=0$ of $S\left(p_{0}\right)$ is degenerate, and $n \geq 3$.

2.3. The $2 \mathrm{D}$ case. If $n=2$, then the order of the conjugate vectors must be one since in the radial direction the derivative of the exponential map is non-zero. Only $(\mathrm{F})$ and $\left(B_{1}\right)$ regular conjugate points are possible among the types listed above. For each $p_{0}$ and $v_{0} \in S\left(p_{0}\right)$, then

Either $N_{p_{0}}\left(v_{0}\right)$ is transversal to $S\left(p_{0}\right)$; or $N_{p_{0}}\left(v_{0}\right)=T_{v_{0}} S\left(p_{0}\right)$

In the first case, $v_{0}$ is of fold type. The second case is more delicate and depends of the order of contact of $N_{p_{0}}\left(v_{0}\right)$ with $S\left(p_{0}\right)$. To be more precise, let $\xi$ be a smooth non-vanishing vector field along $S\left(p_{0}\right)$ so that at each point $v \in S\left(p_{0}\right), \xi$ is collinear with $N_{p_{0}}(v)$. Let $\kappa$ be a smooth function 
on $T_{p_{0}} M$ with a non-zero differential so that $\kappa=0$ on $S\left(p_{0}\right)$. Then $\mathrm{d} \kappa(\xi)$ restricted to $S\left(p_{0}\right)$, has a zero at $v=v_{0}$, see also [12] for this and for the definition below.

Definition 2.1. If this zero is simple, we say that $v_{0}$ is a simple cusp.

Near a simple cusp, the exponential map has the following normal form [12]

$$
y^{1}=\xi^{1}, \quad y^{2}=\xi^{1} \xi^{2}+\left(\xi^{2}\right)^{3} .
$$

Then $\operatorname{det} d_{\xi} \exp _{p_{0}} \xi=\xi^{1}+3\left(\xi^{2}\right)^{2}$ and

$$
\begin{aligned}
& S\left(p_{0}\right)=\left\{\xi^{1}+3\left(\xi^{2}\right)^{2}=0\right\}, \quad N_{p_{0}}\left(v_{0}\right)=\operatorname{span}\left\{\partial / \partial \xi^{2}\right\}, \\
& \Sigma\left(p_{0}\right)=\left\{y^{1}=-3 t^{2}, y^{2}=-2 t^{3},|t| \ll 1\right\} .
\end{aligned}
$$

Note that away from $\left(\xi^{1}, \xi^{2}\right)=(0,0)$, we have a fold since then the first alternative of $(2.7)$ holds. Such a cusp is clearly visible in our numerical experiments in Figure 5.

Regardless of the type of the conjugate vector $v$, the tangent conjugate locus $S(p)$ to any $p$ is a smooth curve. This follows form [40] since the order of any conjugate vector is always one. Its image $\Sigma(p)$ under the exponential map however is locally a smooth curve if $v$ is a fold, a point, if $v$ is of $\left(B_{1}\right)$ type, and a curve with a possible singularity at $v$ when the second alternative in (2.7) holds.

\section{The geOdesic X-RAY transform as AN FIO}

The properties of $X$ as an FIO have been studied in [13], including those of the restricted X-ray transform, in the framework of Guillemin [14] and Guillemin and Stenberg [15]. We will recall the results in [13] with some additions.

3.1. The general $n$ dimensional case. Assume first $n:=\operatorname{dim} M \geq 2$. We extend the manifold and the metric to some neighborhood of $M$. We will study $X$ restricted to geodesics in some open set $\mathcal{M}_{0}$ of geodesics with endpoints outside $M$. It is clear (and shown below) that $\mathcal{M}_{0}$ is a manifold. The set of all geodesics $\mathcal{M}$ might not be, if there are trapped ones. We assume first the following

$$
\gamma(t) \in \mathcal{M}_{0} \Longrightarrow \gamma(-t) \notin \mathcal{M}_{0}
$$

This condition simplifies the exposition. In fact later, we study geodesic manifolds for which the opposite holds, and the general case can be considered as a union of the two. The reason for (3.1) is to guarantee that for any $\gamma \in \mathcal{M}_{0}$, each element in $N^{*} \gamma$ is conormal to exactly one geodesic in $\mathcal{M}_{0}$. Let $M_{0}$ be the points on the geodesics in $\mathcal{M}_{0}$, in the interior of $M$. We will study $X$ acting on distributions $f$ supported in $\mathcal{M}_{0}$. In particular, this covers the case of geodesics in some small enough neighborhood of a fixed geodesic $\gamma_{0}$ as shown in Figure 1.

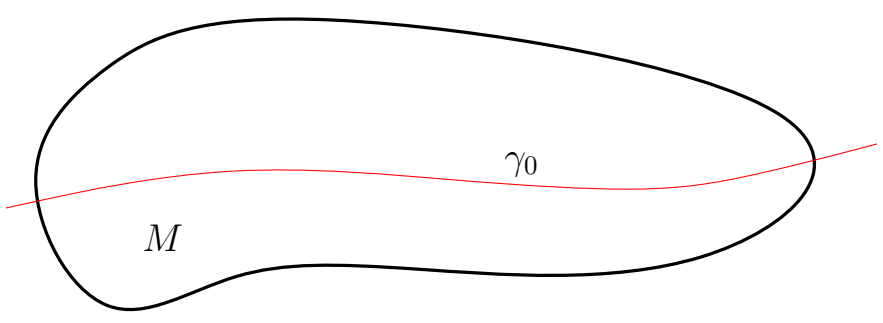

Figure 1. The manifold $M$ and a fixed geodesic $\gamma_{0}$. 
To parameterize the (directed) geodesics near some $\gamma_{0}$, we choose a small oriented hypersurface $H$ intersecting $\gamma_{0}$ transversally. It can be a neighborhood of $\partial M$ near $\gamma(a)$ or $\gamma(b)$ if $\gamma_{0}$ hits $\partial M$ transversely at that particular end. Let $\mathrm{d} \mathrm{Vol}_{H}$ be the induced measure in $H$, and let $\nu$ be a smooth unit normal vector field on $H$ consistent with the orientation of $H$. Let $\mathcal{H}$ consist of all $(p, \theta) \in S M$ with the property that $p \in H$ and $\theta$ is not tangent to $H$, and positively oriented, i.e., $\langle\nu, \theta\rangle>0$. Introduce the measure $\mathrm{d} \gamma=\langle n, \theta\rangle \mathrm{d} \operatorname{Vol}_{H}(p) \mathrm{d} \sigma_{p}(\theta)$ on $\mathcal{H}$. Then one can parametrize all geodesics intersecting $H$ transversally by their intersection $p$ with $H$ and the corresponding direction, i.e., by elements in $\mathcal{H}$. An important property of $\mathrm{d} \gamma$ is that it introduces a measure on that geodesics set that is invariant under a different choice of $H$ by the Liouville Theorem, see e.g., [31].

We can project $\mathcal{H}$ onto the unit ball bundle $B H$ by projecting orthogonally each $\theta$ as a above to $T_{p} H$. The measure $\mathrm{d} \mu$ in this representation becomes the induced measure on $T H$, and in particular, the factor $\langle n, \theta\rangle$ (which does not even make sense in this representation) disappears. We think of $B H$ as a chart for the $2 n-2$ dimensional geodesic manifold $\mathcal{M}_{0}$ (near $\gamma_{0}$ ). Then $B^{*} H$ (which we identify with $B H$ ) has a natural symplectic form, that of $T^{*} H$. We refer to [13] for an invariant definition of $\mathcal{M}_{0}$, in fact, they did that in two ways. It also has a natural volume form, which is invariantly defined on $\mathcal{M}_{0}$. Tangent vectors to $\mathcal{M}_{0}$ can naturally be identified with the Jacobi fields modulo the two trivial ones, $\dot{\gamma}(t)$ and $t \dot{\gamma}(t)$.

We view $X$ as the following (continuous) map

$$
X: C_{0}^{\infty}\left(M_{0}\right) \rightarrow C^{\infty}\left(\mathcal{M}_{0}\right) .
$$

The Schwartz kernel of $X$ is clearly a delta type of distribution which indicates that it is also a (conormal) Lagrangian distribution, and therefore $X$ must be an FIO. To describe it in more detail, we use the double fibration point of view of $[11,16]$. Let

$$
Z_{0}=\left\{(\gamma, x) \in \mathcal{M}_{0} \times M_{0} ; x \in \gamma\right\}
$$

be the point-geodesic relation with $\pi_{\mathcal{M}}: Z_{0} \rightarrow \mathcal{M}_{0}$ and $\pi_{M}: Z_{0} \rightarrow M_{0}$ being the natural left and right projections. It follows from the analysis below that $Z_{0}$ is smooth of dimension $2 n-1$. Then

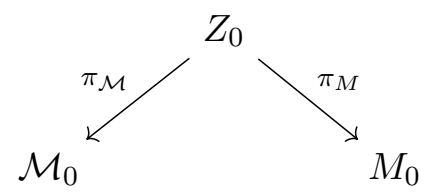

is a double fibration in the sense of [11]. Note that we switched left and right here compared to some other works in order to get a canonical relation later with complies with the notational convention in [19]. To get the microlocal version of that, following Guillemin [14], let

$$
N^{*} Z_{0}=\left\{(\gamma, \Gamma, x, \xi) \in T^{*}\left(\mathcal{M}_{0} \times M_{0}\right) \backslash 0 ;(\gamma, \Gamma)=0 \text { on } T_{(x, \gamma)} Z_{0}\right\}
$$

be the conormal bundle of $Z_{0}$. It is a Lagrangian submanifold of $T^{*}\left(\mathcal{M}_{0} \times M_{0}\right)$, and the associated canonical relation is given by the "twisted" version of $N^{*} Z_{0}$ :

$$
C=N^{*} Z_{0}^{\prime}=\left\{(\gamma, \Gamma, x, \xi) ;(\gamma, \Gamma, x,-\xi) \in N^{*} Z_{0}\right\}
$$

We then have the microlocal version of the diagram above:

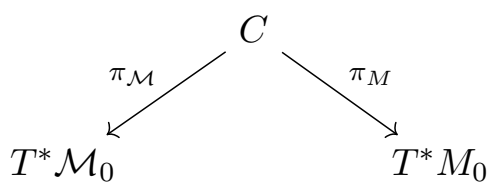


where now $\pi_{\mathcal{M}}$ and $\pi_{M}$ denote the projections indicated above. It is easy to see that their ranges do not include the zero sections.

The weighted X-ray transform $X$ then has a Schwartz kernel $\kappa \delta_{Z_{0}}$ defined by

$$
\left\langle\kappa \delta_{Z_{0}}, \phi\right\rangle=\int_{\mathcal{M}_{0}}\left(\int_{\mathbf{R}} \kappa(\gamma(s), \dot{\gamma}(s)) \phi(\gamma(s), \gamma) \mathrm{d} s\right) \mathrm{d} \gamma
$$

One can think of $\kappa$ as a function defined on $Z_{0}$. Then $\kappa \delta_{Z_{0}}$ is a distribution conormal to $Z_{0}$, see $\left[18\right.$, section 18.2] in the class $I^{-n / 4}\left(M_{0} \times \mathcal{M}_{0}, Z_{0}\right)$. Therefore,

$$
X: C_{0}^{\infty}\left(M_{0}\right) \longrightarrow C_{0}^{\infty}\left(\mathcal{M}_{0}\right)
$$

is an FIO of order $-n / 4$ associated with the Lagrangian $N^{*} Z_{0}$, which can be extended to distributions, as well. Its canonical relation is given by $C$ above.

Let $H$ be as above and fix some local coordinates $y$ on $H$; and let $\eta$ be the dual variable. We denote below by $\gamma(y, \eta, t)$ the geodesic issued from $(y, \tilde{\eta})$, where $\tilde{\eta}$ is unit and has projection $\eta$ on $T H$ (with a fixed orientation). In those coordinates, the manifold $Z_{0}$ consist of all $(y, \eta, x) \in B H \times M$ with the property that $x=\gamma(y, \eta, t)$ for some $t$. We can think of this as a parametric representation

$$
(y, \eta, t) \longmapsto(y, \eta, \gamma(y, \eta, t)) .
$$

Then the map (3.4) has Jacobian

$$
J:=\left(\begin{array}{ccc}
\mathrm{Id} & 0 & 0 \\
0 & \mathrm{Id} & 0 \\
\partial x / \partial y & \partial x / \partial \eta & \partial x / \partial t
\end{array}\right) .
$$

Here, $\partial x / \partial y=\left\{\partial x^{i} / \partial y^{\alpha}\right\}$ with $i=1, \ldots, n$ and $\alpha=1, \ldots, n-1, x=\gamma(y, \eta, t)$, and similarly for $\partial x / \partial \eta$. The identity operators above are in $\mathbf{R}^{n-1}$. Since the bottom right element, the tangent to the geodesic $\gamma(y, \eta, t)$ is never zero, we get that the $(3 n-2) \times(2 n-1)$ matrix $J$ has maximal rank $2 n-1$. In particular, this shows that $Z_{0}$ is a smooth manifold of dimension $2 n-1$.

The conormal bundle $N^{*} Z_{0}$ is at any point of $Z_{0}$ the space conormal to the range of $J$. Denote by $(\hat{y}, \hat{\eta}, \xi)$ the dual variables of $(y, \eta, x)$. Then $(y, \eta, \hat{y}, \hat{\eta}, x, \xi) \in C=N^{*} Z_{0}^{\prime}$ if and only if $(y, \eta, x) \in C$ and

$$
\xi_{i} \dot{\gamma}^{i}(y, \eta, t)=0, \quad \xi_{i} \frac{\partial \gamma^{i}(y, \eta, t)}{\partial y^{\alpha}}-\hat{y}_{\alpha}=0, \quad \xi_{i} \frac{\partial \gamma^{i}(y, \eta, t)}{\partial \eta^{\alpha}}-\hat{\eta}_{\alpha}=0, \quad \alpha=0, \ldots, n-1 .
$$

The first condition says that $\xi$ is conormal to the geodesic issued from $(y, \eta)$, which is consistent with the fact that we can only hope to recover conormal singularities at the geodesics involved in the X-ray transform. In particular, we get

$$
\pi_{M}(C)=\cup_{\gamma \in \mathcal{M}} N^{*} \gamma:=V .
$$

What we see immediately is that we have the inclusion $\subset$ above. On the other hand, given $(x, \xi) \in$ $V$, there is a geodesic in $\mathcal{M}_{0}$ normal to $(x, \xi)$ by the definition of $V$. Then we can compute $\hat{y}$ and $\hat{\eta}$ as above, see also the $2 \mathrm{D}$ case below. Notice that we have an $(n-2)$-dimensional manifold of geodesics normal to $(x, \xi)$; only one, undirected, if $n=2$. In the latter case, we may have two directed ones. In particular this implies that the rank of $\mathrm{d} \pi_{M}$ is maximal and equal to $2 n$ but since $\mathrm{d} \pi_{M}$ maps locally $\mathbf{R}^{3 n-2}$ to $\mathbf{R}^{2 n}$, it has an $n-2$ dimensional kernel, if $n \geq 3$, and is an isomorphism when $n=2$.

The next two equations in (3.5) say that the projection of $\xi$ (identified with a vector by the metric) to any non-trivial Jacobi field, at the point $x=\gamma(y, \eta, t)$, is given. Set

$$
\mathcal{V}:=\pi_{\mathcal{M}}(C)
$$


The projection $\pi_{\mathcal{M}}$ maps the $3 n-2$ dimensional $C$ to the $4 n-4$ dimensional $T^{*} \mathcal{M}_{0}$. We want to find out when this allows us to recover $t$, and therefore, $x$. Assume that we have two different values $t=t_{1}$ and $t=t_{2}$ of $t$, at which (3.5) holds with the so given $(y, \eta, \hat{y}, \hat{\eta})$ with $\left(x_{1}, \xi^{1}\right)$ and $\left(x_{2}, \xi^{2}\right)$, respectively. Consider all non-trivial Jacobi fields vanishing at $t_{1}$. They form a linear space of dimension $n-1$. Their projections to $\xi^{1}$ at $t_{1}$ vanish in a trivial way. By (3.5) and by our assumptions, their projections to $\xi^{2}$ at $t_{2}$ must vanish as well. Since $\xi^{2}$ is not tangent to the geodesic $\gamma(y, \eta, t)$, we get that at $t_{2}$, those Jacobi fields form a subspace of dimension $n-2$. Therefore, a certain non-trivial linear combination vanishes there, which means that there is a Jacobi field vanishing at both $t_{1}$ and $t_{2}$. Then the corresponding $x_{1}$ and $x_{2}$ are conjugate points, as a consequence of our assumption of lack of injectivity.

This argument also proves that $\mathrm{d} \pi_{\mathcal{M}}$ is injective as well. Indeed, locally, there are no conjugate points. The problem then can be reduced to showing that $t=t_{1}$ is a simple root of the equations (3.5) with $(y, \eta, \hat{y}, \hat{\eta})$ given, which follows from the fact that the zeros of Jacobi fields are always simple.

On the other hand, assume that there are no conjugate points along the geodesic $\exp _{y}(t \eta)$. Then any Jacobi field vanishing at $t=t_{1}$ would be nonzero for any other $t$. Those (non-trivial) Jacobi fields span an $(n-1)$-dimensional linear space as above at any $t \neq t_{1}$. On the other hand, they are all perpendicular to $\xi^{1}$ at $t_{1}$. Since $\xi^{1} \neq 0$, we get a contradiction.

Therefore, $\pi_{\mathcal{M}}$ is injective if and only if there are no conjugate points along the geodesics in $\mathcal{M}_{0}$. In particular, it is always locally injective. Moreover, $\mathrm{d} \pi_{\mathcal{M}}$ is injective as well.

The X-ray transform $X$ is said to satisfy the Bolker condition [12] if $\pi_{\mathcal{M}}$ is an injective immersion. Then $X^{*} X$ is a $\Psi D O$ of order -1 . It is elliptic at $(x, \xi)$ if and only if $\kappa(x, \theta) \neq 0$ for at least one $\theta$ with $\langle\theta, \xi\rangle=0$, see, e.g., $[10,35]$. Then one can recover singularities conormal to all geodesics over which we integrate by elliptic regularity. The analysis above yields the following.

Proposition 3.1. The Bolker condition is satisfied for $Z_{0}$ if and only if none of the geodesics in $\mathcal{M}_{0}$ has conjugate points.

An indirect indication of the validity of this proposition is the fact that $X^{*} X$ is a $\Psi D O$ if and only if there are no conjugate points, as mentioned above. The latter however was proved by analyzing the Schwartz kernel of $X^{*} X$ directly, instead of composing $X^{*}$ and $X$ as FIOs. In the more difficult case of the restricted X-ray transform $\left(\mathcal{M}_{0}\right.$ is a submanifold of $\mathcal{M}$ of the same dimension as $M_{0}$, when $n \geq 3$ ), the Bolker condition can be violated even if there are no conjugate points, for examples for the Euclidean metric [13].

We summarize the results so far, most of them due to $[13,14]$, in the following. Let $\mathcal{M}_{0}, M_{0}$, $C$ be as above. We recall that the zero subscript indicates that we work in an open subset of geodesics.

Theorem 3.1. $X$ is a Fourier Integral Operator in the class $I^{-\frac{n}{4}}\left(\mathcal{M}_{0} \times M_{0}, C^{\prime}\right)$. It satisfies the Bolker condition if and only if the geodesics in $\mathcal{M}_{0}$ have no conjugate points. In the latter case, $X^{*} X$ is a $\Psi D O$ of order -1 in $M_{0}$.

We also recall the result in [37] showing that if the conjugate points are of fold type, $X^{*} X$ has a canonical relation constituting of the following non-intersecting canonical relations: the diagonal (a $\Psi$ DO part) and $N^{*} \Sigma^{\prime}$, where $\Sigma$ is the conjugate locus defined as the pairs of all conjugate points, a smooth manifold in that case, see [13].

3.2. The 2-dimensional case. Assume now $n:=\operatorname{dim} M=2$. In this case, the three manifolds in the diagram (3.3) have the same dimension, 4. A natural question is whether $\pi_{\mathcal{M}}$ and $\pi_{M}$ are 
diffeomorphisms, local or global. The analysis of the $n$-dimensional case answers this already but we will make this more explicit below.

We introduce the scalar Jacobi fields $a$ and $b$ following [24] below. Introduce the notation $v^{\perp}$, where for a given vector $v$, we define the covector $v^{\perp}$ by $v_{i}^{\perp}:=\mathcal{R}_{i j} v^{j}$, where

$$
\mathcal{R}_{i j}=\sqrt{\operatorname{det} g}\left(\begin{array}{cc}
0 & 1 \\
-1 & 0
\end{array}\right) .
$$

Note that $v^{\perp}$ has the same length as the vector $v$ and is conormal to $v$. The inverse map is then given by $\xi_{\perp}^{i}=\sum_{j} \mathcal{R}_{i j}^{-1} \xi_{j}$. If we think of $v \mapsto v^{\perp}$ as a rotation by -90 degrees, then $\xi \mapsto \xi_{\perp}$ is a rotation by 90 degrees.

With $H$ as above (now, a curve transversal to $\gamma_{0}$ ), every Jacobi field along any fixed geodesic $\gamma$ close to $\gamma_{0}$ is a linear combination $\hat{y} a(y, \eta, t)+\hat{\eta} b(y, \eta, t)$, where $(\hat{y}, \hat{\eta})$ are the initial conditions. As before, $(y, \eta) \in B H$. The first of the conditions (3.5) say that for such a point in $C$, we have $\xi=\lambda \dot{\gamma}^{\perp}$ with some $\lambda \neq 0$. The last two equations imply $\lambda a=\hat{y}, \lambda b=\hat{\eta}$ where

$$
a(y, \eta, t)=\frac{\partial \gamma^{i}}{\partial y} \dot{\gamma}_{i}^{\perp}, \quad b(y, \eta, t)=\frac{\partial \gamma^{i}}{\partial \eta} \dot{\gamma}_{i}^{\perp} .
$$

The functions $a$ and $b$ are the projections of the Jacobi fields $\partial \gamma^{i} / \partial y$ and $\partial \gamma^{i} / \partial \eta$ to $\dot{\gamma}^{\perp}$. They solve the scalar Jacobi equation

$$
\ddot{a}+K a=0, \quad \ddot{b}+K b=0,
$$

where $K$ is the Gauss curvature, with linearly independent initial conditions. Then

$$
C=\left\{\left(y, \eta, \lambda a(y, \eta, t), \lambda b(y, \eta, t), \gamma(y, \eta, t), \lambda \dot{\gamma}^{\perp}(y, \eta, t)\right) \mid(y, \eta) \in B H, \lambda \neq 0, t \in \mathbf{R}\right\},
$$

compare with (3.3). Clearly, $\operatorname{dim} C=4$, therefore, all manifolds in the diagram (3.3) are of the same dimension, 4 .

The Bolker condition which we analyzed above says that $\pi_{\mathcal{M}}$ is an injective immersion if and only if there are no conjugate points along the geodesics in $\mathcal{M}_{0}$. In particular, this is true near $\gamma_{0}$ if there are no conjugate points on $\bar{\gamma}_{0}$. We will prove this again in this $2 \mathrm{D}$ situation. Fix $(y, \eta, \hat{y}, \hat{\eta})$ with $(\hat{y}, \hat{\eta}) \neq 0$. We want to see first if the time $t$ for which

$$
\lambda a(y, \eta, t)=\hat{y}, \quad \lambda b(y, \eta, t)=\hat{\eta}
$$

is unique. Consider the scalar non-zero Jacobi field $c(y, \eta, t):=\hat{y} b(y, \eta, t)-\hat{\eta} a(y, \eta, t)$ that vanishes when (3.9) holds. The problem is reduced to showing uniqueness of the solution to $c(y, \eta, t)=0$ with respect to $t$. If there are two solutions however, then they correspond to conjugate points. This proves the injectivity of the projection $\pi_{\mathcal{M}}$ in this case. The injectivity of differential of $\pi_{\mathcal{M}}$ follows from the fact that $\mathrm{d} c / \mathrm{d} t \neq 0$ when $c=0$. Since $\operatorname{dim} T^{*} \mathcal{M}_{0}=\operatorname{dim} C=4$, we get that $\pi_{\mathcal{M}}$ is actually a local diffeomorphism. It is global, from $C$ to $\mathcal{V}$, assuming no conjugate points along any geodesic in $\mathcal{M}_{0}$.

We show now that $\pi_{M}: C \rightarrow V$ is a diffeomorphism, see (3.6). For $(x, \xi) \in N^{*} \gamma$, for some $\gamma \in \mathcal{M}$, let $\gamma_{x, v}$ be the unique, by (3.1), geodesic with the unit $v$ so that $v= \pm(\xi /|\xi|)_{\perp}$. Without loss of generality we may assume that the sign above is positive. Let $y \in H$ be the point where it hits $H$ for the first time, $t$, and let $\eta$ be the projection of the direction at $y$ to $T H$. Then $(y, \eta, t)$ depend smoothly on $(x, \xi)$ as a consequence of the assumption that $\gamma_{0}$ hits $H$ transversely. Thus the inverse of $\pi_{M}$ is given by

$$
\pi_{M}^{-1}:(x, \xi) \mapsto(y, \eta, \lambda a(y, \eta, t), \lambda b(y, \eta, t), x, \xi), \quad y=y(x, \xi), \eta=\eta(x, \xi), t=t(x, \xi)
$$

with the last three functions defined as above. So $\pi_{M}$ is a local diffeomorphism. If the opposite to (3.1) holds, then $\pi_{M}$ is a double cover. 
Combining this with the previous paragraph, we get in particular that $\mathcal{C}(x, \xi)=(y, \eta, \lambda a, \lambda b)$ given by $\mathcal{C}=\pi_{\mathcal{M}} \circ \pi_{M}^{-1}$ is a local diffeomorphism.

We summarize those results below. Recall that $\mathcal{M}_{0}$ is an open subset of geodesics and that $M_{0} \subset M$ consist of the interior point on those geodesics, and that $X$ is restricted to $\mathcal{M}_{0} \times M_{0}$.

Theorem 3.2. Let $\operatorname{dim} M=2$. Then under the assumptions in this section,

(a) $X$ is an FIO of order $-1 / 2$ associated with the canonical relation $C$ given by (3.8), which is a graph of the canonical map $\mathcal{C}$ described above.

(b) $\mathcal{C}$ is a local diffeomorphism. It is a global one, from $V$ to $\mathcal{V}$ if and only if there are no conjugate points on the geodesics in $\mathcal{M}_{0}$.

(c) If there are no conjugate points on the geodesics in $\mathcal{M}_{0}, X$ is elliptic at $(x, \xi)$ if and only if $\kappa(x, v) \neq 0$ for $v$ such that $v^{\perp}$ is collinear with $\xi$.

Conjugate points destroy the injectivity of $\mathcal{C}$, while a violation of condition (3.1) (assumed above) makes $\mathcal{C}$ 1-to-2.

Notice also that regardless of existence or not of conjugate points, $X: H^{s}\left(M_{0}\right) \rightarrow H^{s+1 / 2}\left(\mathcal{M}_{0}\right)$ continuously because one can use a partition of unity and reduce the problem to the case of no conjugate points, see also [37]. Ellipticity of $X$ is understood in the sense of $[19, \S 25.3]$.

\section{Cancellation of singularities and instability}

We are ready now to prove a stronger version of the cancellation of singularities of $X$ when $\operatorname{dim} M=2$. First, we will prove that we have a cancellation of infinite order. Second, the type of the conjugate locus will play no role at all. More precisely, since $X$ maps $H^{s-1 / 2}$ to $H^{s}$ locally, resolution of singularities without loss of derivatives would mean $X f \in H^{s}\left(\gamma_{0}, \Gamma^{0}\right) \Longrightarrow f \in H^{s-1 / 2}\left(x_{0}, \xi^{0}\right)$ for any $\left(\gamma_{0}, \Gamma^{0}, x_{0}, \xi^{0}\right) \in C$. We proved in [37] that this is not true if the conjugate points are of fold type, and there is an infinite dimensional space of distributions, for which $f \in H^{s-1 / 2}\left(x_{0}, \xi^{0}\right)$ but $X f \in H^{s+1 / 4}\left(\gamma_{0}, \Gamma^{0}\right)$, and this is true actually in open conic neighborhoods of those points. This is a loss of at least of $1 / 4$ derivatives, even if we can recover $\mathrm{WF}(f)$ in another Sobolev space. We show below that we have actually loss of all derivatives, and the type of the conjugate points does not matter.

Assume from now on that $\mathcal{M}_{0}$ is a small neighborhood of some $\gamma_{0}$. Then the set $V$ has two natural disjoint components, corresponding to the choice of the orientation of the normals to the geodesics. In the representation (3.8), this corresponds to the choice of the sign of $\lambda$. Assume the convention that $\lambda>0$ corresponds to the positive orientation. Then

$$
V=V_{-} \cup V_{+}, \quad \mathcal{V}=\mathcal{V}_{-} \cup \mathcal{V}_{+} ; \quad \mathcal{V}_{ \pm}:=\mathcal{C}\left(V_{ \pm}\right)
$$

To understand better what $\mathcal{C}\left(p_{1}, \xi^{1}\right)=\mathcal{C}\left(p_{2}, \xi^{2}\right)$ means, observe first that the latter is equivalent to the following. The points $p_{1}$ and $p_{2}$ belong to the same geodesic $\gamma(y, \eta, t)$, i.e., $p_{1}=\gamma\left(t_{1}, y, \eta\right)$, $p_{2}=\gamma\left(t_{2}, y, \eta\right)$. Next,

$$
\lambda_{1} a\left(t_{1}, y, \eta\right)=\lambda_{2} a\left(t_{2}, y, \eta\right), \quad \lambda_{1} b\left(t_{1}, y_{0}, \eta\right)=\lambda_{2} b\left(t_{2}, y_{0}, \eta\right),
$$

and

$$
\xi^{1}=\lambda_{1} \dot{\gamma}^{\perp}\left(t_{1}, y, \eta\right), \quad \xi^{2}=\lambda_{2} \dot{\gamma}^{\perp}\left(t_{2}, y, \eta\right) .
$$

In what follows, we drop the dependence on $(y, \eta)$. The Wronskian $W(t):=a(t) \dot{b}(t)-\dot{a}(t) b(t)$ is independent of $t$ and therefore equal to its initial condition $W(0) \neq 0$. Consider the Jacobi field $c(t):=a\left(t_{1}\right) b(t)-b\left(t_{1}\right) a(t)$ (as we did above). Then $c\left(t_{1}\right)=c\left(t_{2}\right)=0$. We have

$$
\dot{c}\left(t_{1}\right)=W(0), \quad \dot{c}\left(t_{2}\right)=\left(\lambda_{2} / \lambda_{1}\right) W(0) .
$$




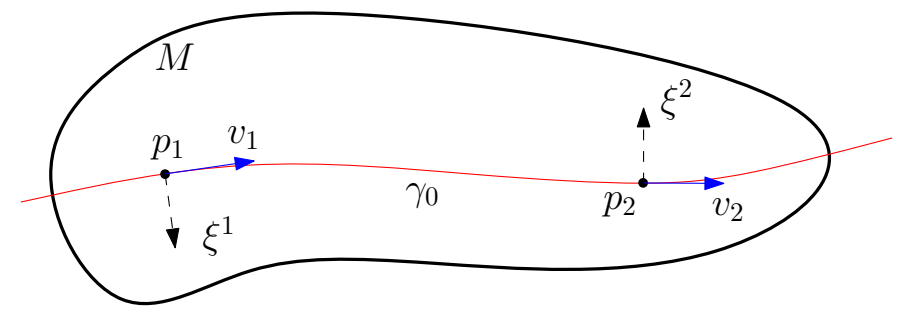

Figure 2. Singularities that cancel; $p_{1}$ and $p_{2}$ are conjugate and there is an even number of conjugate points (or none) between $p_{1}$ and $p_{2}$. Next, $\left(p_{2}, \xi^{2}\right)=\mathcal{C}_{21}\left(p_{1}, \xi^{1}\right)$, see (4.3).

Therefore,

$$
\lambda_{1}=\lambda \dot{c}\left(t_{1}\right), \quad \lambda_{2}=\lambda \dot{c}\left(t_{2}\right), \quad \lambda \neq 0 .
$$

We therefore proved the following.

Theorem 4.1. $\mathcal{C}\left(p_{1}, \xi^{1}\right)=\mathcal{C}\left(p_{2}, \xi^{2}\right)$ if and only if there is a geodesic $[0,1] \rightarrow \gamma \in \mathcal{M}_{0}$ joining $p_{1}$ and $p_{2}$ (i.e. $\gamma(0)=p_{1}$ and $\gamma(1)=p_{2}$ ) so that

(a) $p_{1}$ and $p_{2}$ are conjugate to each other, along $\gamma$,

(b) $\xi^{1}=\lambda \dot{J}(0), \xi^{2}=\lambda \dot{J}(1), \lambda \neq 0$, where $J(t)$ is the unique non-trivial, up to multiplication by a constant, Jacobi field with $J(0)=J(1)=0$.

Of course, (b) is equivalent to saying that $\xi^{1}=\lambda \dot{c}(0) \dot{\gamma}^{\perp}, \xi^{2}=\lambda \dot{c}(1) \dot{\gamma}^{\perp}, \lambda \neq 0$, where $c(t)$ is the unique non-trivial, up to multiplication by a constant, Jacobi field with $c(0)=c(1)=0$. In particular, $\xi^{1}$ and $\xi^{2}$ are conormal to $\gamma$. This generalizes a result in [37] proved there under the assumption that the conjugate points are of fold type.

From now on, we fix $\left(p_{1}, \xi^{1}\right)$ and $\left(p_{2}, \xi^{2}\right)$ so that $\mathcal{C}\left(p_{1}, \xi^{1}\right)=\mathcal{C}\left(p_{2}, \xi^{2}\right)$. Let $V^{1}=V_{-}^{1} \cup V_{+}^{1}$ be a small conic neighborhood of $\left(p_{1}, \xi^{1}\right)$, and let $V^{2}=V_{-}^{2} \cup V_{+}^{2}$ be a small conic neighborhood of $\left(p_{2}, \xi^{2}\right)$. Note that the signs of $\dot{c}(0)$ and $\dot{c}(1)$ in Theorem 4.1 above are the opposite if the number $m$ of conjugate points between $p$ and $q$ is even (or zero); and the signs are equal otherwise. Let $\epsilon=(-1)^{m}$. By shrinking those neighborhoods a bit, we can assume that $\mathcal{C}\left(V_{ \pm}^{1}\right)=\mathcal{C}\left(V_{\mp \epsilon}^{2}\right)=: \mathcal{V}_{ \pm}$. Let $\mathcal{C}_{k}:=\left.\mathcal{C}\right|_{V^{k}}, k=1,2$, where, somewhat incorrectly, $\pm(-1)^{m}= \pm$, if $m$ is even, and $\pm(-1)^{m}=\mp$. Set

$$
\mathcal{C}_{21}:=\mathcal{C}_{2}^{-1} \circ \mathcal{C}_{1}: V^{1} \rightarrow V^{2} .
$$

Then $\mathcal{C}_{21}$ is a canonical relation itself, and is a diffeomorphism. Also, $\mathcal{C}_{21}: V_{ \pm}^{1} \rightarrow V_{\mp \varepsilon}^{2}$.

Next theorem extends the corresponding result in [37] from the case of fold conjugate points (in any dimension) to any type of conjugate points (in two dimensions). It relates the canonical relation $\mathcal{C}_{21}$ directly to the geometry of the conjugate locus.

\section{Theorem 4.2.}

(a) $\mathcal{C}_{21}: V^{1} \rightarrow V^{2}$ is a diffeomorphism.

(b) $\mathcal{C}_{21}:\left(p_{1}, \xi^{1}\right) \rightarrow\left(p_{2}, \xi^{2}\right)$ also admits the characterization of Theorem 4.1.

The following theorem describes the microlocal kernel of $X$ in this setup.

Theorem 4.3. Let $\kappa\left(p_{2}, \xi^{2}\right) \neq 0$. Then there exists an FIO $F_{21}$ of order zero with canonical relation $\mathcal{C}_{21}$ with the following property. Let $f_{k} \in \mathcal{E}^{\prime}\left(M_{0}\right)$ with $\mathrm{WF}\left(f_{k}\right) \subset V^{k}, k=1,2$, with $V^{1}, V^{2}$ small enough. Then

$$
X\left(f_{1}+f_{2}\right) \in H^{s}(\mathcal{V})
$$


if and only if

$$
f_{2}+F_{21} f_{1} \in H^{s-1 / 2}\left(V^{2}\right) .
$$

The FIO $F_{21}$ is elliptic if and only if $\kappa\left(p_{1}, \xi^{1}\right) \neq 0$.

Clearly, under the ellipticity assumptions above, we can swap the indices 1 and 2 to obtain

$$
X\left(f_{1}+f_{2}\right) \in H^{s}(\mathcal{V}) \Longleftrightarrow f_{2}+F_{21} f_{1} \in H^{s-1 / 2}\left(V^{2}\right) \quad \Longleftrightarrow f_{1}+F_{12} f_{2} \in H^{s-1 / 2}\left(V^{1}\right),
$$

where $F_{12}=F_{21}^{-1}$ (microlocally).

Proof. Let $X_{k}$ be $X$, restricted to distributions with wave front sets in $V_{k}, k=1,2$. Then $X f=$ $X_{1} f_{1}+X_{2} f_{2}$. We proved above that $X_{k}$ are FIOs with canonical relations $\mathcal{C}_{k}, k=1$, 2; elliptic, if $\kappa\left(p_{k}, \xi^{k}\right) \neq 0$. Then an application of the parametrix $X_{2}^{-1}$ to $X f$ completes the proof. In particular, we get

$$
F_{12}=X_{2}^{-1} X_{1}, \quad F_{21}=X_{1}^{-1} X_{2} .
$$

The theorem implies that we cannot resolve the singularities from the singularities of $X f$ near $\gamma_{0}$.

Corollary 4.1. Given $f_{1} \in \mathcal{E}^{\prime}\left(M_{0}\right)$ with $\mathrm{WF}\left(f_{1}\right) \subset V^{1}$, there exists $f_{2} \in \mathcal{E}^{\prime}\left(M_{0}\right)$ with $\mathrm{WF}\left(f_{2}\right) \subset V^{2}$ so that $X\left(f_{1}+f_{2}\right) \in C^{\infty}(\mathcal{V})$.

In other words, we can cancel any singularities close to $\left(p_{1}, \xi^{1}\right)$ with a suitable chosen "annihilator" with a wave front set near $\left(p_{2}, \xi^{2}\right)$.

Remark 4.1. The results so far can be easily extended to $f=f_{1}+\cdots+f_{m}$, where $f_{j}$ are microlocally supported near $N^{*} \gamma$ over neighborhoods of $p_{1}, \ldots, p_{m}$, conjugate to each other. Then $X f$ is microlocally equivalent to $X_{1} f_{1}+\cdots+X_{m} f_{m}$, with all $X_{j}$ elliptic FIOs associated to canonical graphs of diffeomorphisms, if $\kappa \neq 0$. Given $f_{1}, \ldots, f_{m}$ with the exception of $f_{i}$ with $i$ fixed, one can find $f_{i}$ which annihilates all singularities by simply inverting $X_{i}$.

So far, we assumed that we know $X f$ locally, and in that in particular (3.1) holds. If $\kappa(x, \theta)$ is an even function of $\theta$, replacing $\gamma(t)$ by $\gamma(-t)$ provides the same information. This would also be the case if the matrix in (5.1) has rank one. We can formulate the following global results. Part (a) of the theorem below is essentially known, see, e.g., [30, 35].

Theorem 4.4. Let $\left(M_{1}, g\right)$ be a Riemannian manifold and let $M \Subset M_{1}$ be a compact submanifold with boundary. Assume that all geodesics having a common point with $M$ exit $M$ at both ends (i.e., $M$ is non-trapping). Let $\mathcal{M}_{0}$ be an open set of geodesics so that

$$
\cup_{\gamma \in \mathcal{M}_{0}} N^{*} \gamma \supset T^{*} M
$$

Then

(a) If there are no conjugate points on any maximal geodesic in $M_{1}$, then there exists $s_{1}$ so that

$$
\|f\|_{H^{s-1 / 2}\left(M_{1}\right)} \leq C_{s}\|X f\|_{H^{s}\left(\mathcal{M}_{0}\right)}+C_{s}\|f\|_{H^{s_{1}\left(M_{1}\right)}}, \quad \forall s \geq 1 / 2, \quad \forall f \in C^{\infty}(M) .
$$

In particular, if $X$ restricted to $\mathcal{M}_{0}$ is injective on $C_{0}(M)$, then then we have

$$
\|f\|_{H^{s-1 / 2}\left(M_{1}\right)} \leq C\|X f\|_{H^{s}\left(\mathcal{M}_{0}\right)} .
$$

(b) Let $\kappa(x, \theta)=\kappa(x,-\theta)$. If at least one geodesic in $M_{1}$ has conjugate points in the interior of $M$, then the following estimate does not hold, regardless of the choice of $s_{1}, s_{2}, s_{3}$ :

$$
\|f\|_{H^{s_{1}\left(M_{1}\right)}} \leq C\|X f\|_{H^{s_{2}\left(\mathcal{M}_{0}\right)}}+C\|f\|_{H^{s_{3}\left(M_{1}\right)}} .
$$


Remark 4.2. Here, we define the Sobolev spaces on $\mathcal{M}$ via a fixed coordinate atlas. An invariant definition is also possible, for example by identification of $\mathcal{M}$ and a subset of the unit ball bundle $B M_{1}$, where $M_{1} \ni M$ as above.

Remark 4.3. We do not assume convexity here. If a geodesic $\gamma$ has a few disconnected segments in $M$, (b) is still true since we regard the integral $X f(\gamma)$ as one number. On the other hand, if we know the integral over each segment, we may be able to resolve the singularities, if (a) applies to each one, and then (b) may fail.

Remark 4.4. Condition (4.6) says that for each $(x, \xi)$ there is at least one (directed) geodesic through $x$ normal to $\xi$. This, of course, means that if we ignore the direction, all geodesics through $M$ must be in $\mathcal{M}_{0}$. The direction however matters because $\kappa$ is not necessarily even w.r.t. $\theta$. In (c), condition (4.6) simply means that $\mathcal{M}$ must contain all geodesics through $M$.

Remark 4.5. It is easy to prove that under the assumptions in (b), even the weaker conditional type of estimate of the type (4.9), with $\|X f\|_{H^{s_{2}\left(\mathcal{M}_{0}\right)}}$ replaced by $\|X f\|_{H^{s_{2}\left(\mathcal{M}_{0}\right)}}^{\alpha}\|f\|_{H^{s_{4}\left(M_{1}\right)}}^{\beta}, \alpha+\beta=1$, does not hold.

Proof. Under the assumptions of (a), $X$ is an elliptic FIO of order $-1 / 2$ associated to the canonical graph $C$. Given $(x, \xi) \in T^{*} M \backslash 0$, restrict $X$ to a small neighborhood of the geodesic normal to $\xi$ at $x$, which existence is guaranteed by (4.6). Then $\mathcal{C}$ is a diffeomorphism, by Theorem 3.2. It therefore has a parametrix, and (4.7) follows for $P f$ on the left, where $P$ is a $\Psi$ DO of order 0 essentially supported neat $(x, \xi)$. Using a partition of unity, we prove (4.7). Estimate (4.8) follows from (4.6) and a functional analysis argument, see, e.g., [38, Proposition 5.3.1].

Part (b) follows from Theorem 4.3 and [30], for example.

We note that estimate (4.9) can be microlocalized with pseudo-differential cutoffs in an obvious way; and the same conclusion remains.

Properties of $X^{*} X$. The operator $X^{*}$ is often used as a first step of an inversion of $X$, and it was the main object of study in [37]. We show the connection next between the theorems above and the properties of $X^{*} X$.

With the microlocalization above, $X=X_{1}+X_{2}$. Then

$$
X^{*} X=X_{1}^{*} X_{1}+X_{1}^{*} X_{2}+X_{2}^{*} X_{1}+X_{2}^{*} X_{2}
$$

The operators above are FIOs with canonical relations with the following mapping properties: $X_{1}^{*} X_{1}$ and $X_{2}^{*} X_{2}$ are $\Psi$ DOs acting in $V^{1}$ and $V^{2}$, respectively, $X_{1}^{*} X_{2}$ maps $V^{2}$ to $V^{1}$ microlocally, and its adjoint $X_{2}^{*} X_{1}$ maps $V^{1}$ to $V^{2}$ microlocally. If $\kappa\left(p_{2}, \xi^{2}\right) \neq 0$, then $X_{2}^{*} X_{2}$ is elliptic if $V^{2}$ is small enough, and for any $\Psi$ DO $P_{2}$ essentially supported in $V^{2}$, and for $f=f_{1}+f_{2}$ as above, we have

$$
\left(X_{2}^{*} X_{2}\right)^{-1} P_{2} X^{*} X f=f_{2}+\left(X_{2}^{*} X_{2}\right)^{-1} P_{2} X_{2}^{*} X_{1} f_{2},
$$

where the inverses are parametrices, and the equality is modulo smoothing operators applied to $f$. Then we get (4.5) again with

$$
F_{21}=\left(X_{2}^{*} X_{2}\right)^{-1} P_{2} X_{2}^{*} X_{1}
$$

This is natural - we obtained $F_{21}=X_{2}^{-1} X_{1}$ in Theorem 4.3; and one way to construct $X_{2}^{-1}$ is to apply $X_{2}^{*}$ first, and then to apply $\left(X_{2}^{*} X_{2}\right)^{-1}$. In particular, we get the following generalization of one of the main results in [37] to the $2 \mathrm{D}$ case. 
Theorem 4.5. With the notation and the assumptions above,

$$
\begin{array}{ll}
X^{*} X f=A_{1}\left(f_{1}+F_{12} f_{2}\right) & \text { microlocally in } V^{1}, \\
X^{*} X f=A_{2}\left(f_{2}+F_{21} f_{1}\right) & \text { microlocally in } V^{2},
\end{array}
$$

where $A_{1}=X_{1}^{*} X_{1}$ and $A_{2}=X_{2}^{*} X_{2}$ are $\Psi$ DOs with principal symbols

$$
\frac{\pi}{|\xi|}\left(\left|\kappa_{j}\left(x, \xi_{\perp} /|\xi|\right)\right|^{2}+\left|\kappa_{j}\left(x,-\xi_{\perp} /|\xi|\right)\right|^{2}\right),
$$

$j=1,2$, respectively, and $F_{21}, F_{12}$ are the FIOs of Theorem 4.3.

Note that under assumption (3.1), one of the two summands above vanishes.

This theorem explains the artifacts we will get when using $X^{*} X f$ (times an elliptic $\Psi$ DO) as an attempt to recover $f$. Assume that $f_{2}=0$ and we want to recover $f_{1}$ from $X f_{1}$, and assume that $\kappa \neq 0$. We will get the original $f_{1}$ (in $V^{1}$ ) plus the "artifact" $F_{21} f_{1}\left(\right.$ in $V^{2}$ ). This is not a downside of that particular method; by Theorem 4.3, we cannot say which is the original. The true image might be either $f_{1}$ or $F_{21} f_{1}$, or some convex linear combination of the two. All we can recover is $f_{2}+F_{21} f_{1}$, or, equivalently, $f_{1}+F_{12} f_{2}$.

\section{ReCovery of Singularities AND StABILITy FOR NON-DEgenerate Weights. The ATTENUATED TRANSFORM}

5.1. Heuristic arguments. The analysis so far was concerned with whether we can recover $\mathrm{WF}(f)$ near $N^{*} \gamma_{0}$ from the knowledge of the singularities of $X f$ known near $\gamma_{0}$. We allowed for the geodesic set $\mathcal{M}_{0}$ to be not necessarily small but we assumed (3.1). On the other hand, each $(x, \xi) \in T^{*} M \backslash 0$ can be possibly detected by the two directed geodesics (and the ones close to them) through $x$ normal to $\xi$. If $X f$ is known there, we have two equations for each such $(x, \xi)$. Let us say that we are in the situation in the previous section. Instead of the single equation (4.4), we have two equations, each one coming from each direction. We think of this as a $2 \times 2$ system, and if its determinant is not zero, loosely speaking, we could solve it and still recover the singularities.

In the situation in the previous section, let $\mathcal{C}\left(p_{1}, \xi^{1}\right)=\mathcal{C}\left(p_{2}, \xi^{2}\right)$. Let $\left(p_{1}, v_{1}\right)$ and $\left(p_{2}, v_{2}\right)$, respectively be the corresponding unit tangent vectors, pointing in the same direction along the geodesic connecting $p_{1}$ and $p_{2}$. Without loss of generality, we may assume that $\xi^{1} /\left|\xi^{1}\right|=v_{1}^{\perp}$ (the other option is to be $\left.-v_{1}^{\perp}\right)$. Then $\varepsilon \xi^{2} /\left|\xi^{2}\right|=v_{2}^{\perp}$, where $\varepsilon=(-1)^{m}$, as above, where $m$ is the number of conjugate points between $p_{1}$ and $p_{2}$.

Let $X_{+}$and $X_{-}$be $X$ restricted to a neighborhood of $\gamma_{0}$ with a positive and a negative orientation, respectively. Then

$$
X_{+}\left(f_{1}+f_{2}\right) \in C^{\infty}, \quad X_{-}\left(f_{1}+f_{2}\right) \in C^{\infty}
$$

is a $2 \times 2$ system. Heuristically, $\left(p_{1}, v_{1}\right)$ is involved there with weight $\kappa\left(p_{1}, v_{1}\right)$ in the first equation, and with weight $\kappa\left(p_{1},-v_{1}\right)$ in the second one; similarly for $\left(p_{2}, v_{2}\right)$. Therefore, if

$$
\operatorname{det}\left(\begin{array}{cc}
\kappa\left(p_{1}, v_{1}\right) & \kappa\left(p_{2}, v_{2}\right) \\
\kappa\left(p_{1},-v_{1}\right) & \kappa\left(p_{2},-v_{2}\right)
\end{array}\right) \neq 0,
$$

the system should be solvable and we should be able to recover the singularities at those points. We view this as a non-degeneracy condition. Note that a constant weight is degenerate then.

One such case is the attenuated geodesic X-ray transform with a positive attenuation. The weight $\kappa$ then decreases strictly along the geodesic flow. Then $\kappa\left(p_{1}, v_{1}\right)>\kappa\left(p_{2}, v_{2}\right)$, and $\kappa\left(p_{2},-v_{2}\right)>$ $\kappa\left(p_{1},-v_{1}\right)$, and then the determinant is positive, therefore not zero.

Finally, if we want to resolve three singularities placed at three conjugate to each other points $p_{1}, p_{2}, p_{3}$, we would have two equations for three unknowns, and recovery would not be possible. 


\subsection{Recovery of singularities for non-degenerate weights.}

Theorem 5.1. Let $f=f_{1}+f_{2}$ with $\mathrm{WF}\left(f_{k}\right) \subset V^{k}, k=1,2$. If $\kappa \neq 0$, (5.1) holds, and $V^{1}$ and $V^{2}$ are small enough, then $X\left(f_{1}+f_{2}\right) \in H^{s}(\mathcal{V})$ implies $f_{k} \in H^{s-1 / 2}\left(V^{k}\right), k=1,2$.

Proof. We assume first that $\mathrm{WF}\left(f_{1}\right) \subset V_{+}^{1}$; then $\mathrm{WF}\left(f_{2}\right) \subset V_{-\varepsilon}^{2}$. The case $\mathrm{WF}\left(f_{1}\right) \subset V_{-}^{1}$ is similar. All inverses below are parametrices and all equalities hold modulo $C^{\infty}$ in either $\mathcal{V}$ or $V_{-}^{1}$ or $V_{-\varepsilon}^{2}$, depending on the context. With the notation of the previous section, let $X_{+, k}$ be $X_{+}$restricted to a neighborhood of $p_{k}, k=1,2$, and we similarly define $X_{-, k}$. Then (4.4) gives us two equations

$$
X_{+, 1} f_{1}+X_{+, 2} f_{2}=g_{+}:=X_{+} f \in H^{s}(\mathcal{V}), \quad X_{-, 1} f_{1}+X_{-, 2} f_{2}=g_{-}:=X_{-} f \in H^{s}(\mathcal{V})
$$

Since both $\kappa\left(p_{2}, v_{2}\right)$ and $\kappa\left(p_{2},-v_{2}\right)$ are non-zero by assumption, $X_{ \pm, 2}$ are elliptic, and we have

$$
P f_{1}=X_{+, 2}^{-1} g_{+}-X_{-, 2}^{-1} g_{-}, \quad P:=X_{+, 2}^{-1} X_{+, 1}-X_{-, 2}^{-1} X_{-, 1} \text {. }
$$

We have

$$
P=X_{+, 2}^{-1} X_{+, 1}(\operatorname{Id}-Q), \quad Q:=X_{+, 1}^{-1} X_{+, 2} X_{-, 2}^{-1} X_{-, 1} .
$$

The operator $X_{+, 2} X_{-, 2}^{-1}$ is a $\Psi \mathrm{DO}$ on $\mathcal{M}_{0}$. To compute its principal symbol, write

$$
X_{+, 2} X_{-, 2}^{-1}=X_{+, 2}\left(X_{-, 2}^{-1} X_{+, 2}\right) X_{+, 2}^{-1} .
$$

The term $X_{-, 2}^{-1} X_{+, 2}$ in the parentheses in (5.4) is a $\Psi$ DO on $M$. Then the principal symbol of $X_{-, 2}^{-1} X_{+, 2}$ is $\sigma_{2}:=\kappa\left(x, \varepsilon \xi_{\perp} /|\xi|\right) / \kappa\left(x,-\varepsilon \xi_{\perp} /|\xi|\right)$, as it follows from Theorem 4.5, its obvious generalization to operators with different weights, and the construction of $X_{-, 2}^{-1}$ by applying $X_{-, 2}^{*}$ to $X_{-, 2}$ first. Recall that $X_{-, 2}^{-1} X_{+, 2}$ has essential support near $\left(p_{2}, \pm \xi^{2}\right)$. Then by (5.4) and by Egorov's theorem, the principal symbol of $X_{+, 2} X_{-, 2}^{-1}$ is $\sigma_{2} \circ \mathcal{C}_{2}^{-1}$. In a similar way, by Theorem 4.5, the principal symbol of $X_{-, 1} X_{+, 1}^{-1}$ is $\sigma_{1}=\kappa\left(x,-\xi_{\perp} /|\xi|\right) / \kappa\left(x, \xi_{\perp} /|\xi|\right)$.

We can now write, see (5.3),

$$
Q=X_{-, 1}^{-1}\left(X_{-, 1} X_{+, 1}^{-1}\right)\left(X_{+, 2} X_{-, 2}^{-1}\right) X_{-, 1} .
$$

The operator in the first parentheses, $X_{-, 1} X_{+, 1}^{-1}$, has principal symbol $\sigma_{1} \circ \mathcal{C}_{1}^{-1}$. Applying Egorov's theorem again, we get that the principal symbol of $Q$ is

$$
\left[\left(\sigma_{1} \circ \mathcal{C}_{1}^{-1}\right)\left(\sigma_{2} \circ \mathcal{C}_{2}^{-1}\right)\right] \circ \mathcal{C}_{1}=\sigma_{1}\left(\sigma_{2} \circ \mathcal{C}_{21}\right) .
$$

Since $\mathcal{C}_{21}\left(p_{1}, \xi^{1}\right)=\left(p_{2}, \xi^{2}\right)$, at $\left(p_{1}, \xi^{1}\right), Q$ has principal symbol

$$
\sigma_{p}(Q)\left(p_{1}, \xi^{1}\right)=\frac{\left.\kappa\left(p_{1},-\xi_{\perp}^{1} /\left|\xi^{1}\right|\right)\right)}{\kappa\left(p_{1}, \xi_{\perp}^{1} /\left|\xi^{1}\right|\right.} \frac{\left.\kappa\left(p_{2}, \varepsilon \xi_{\perp}^{2} /\left|\xi^{2}\right|\right)\right)}{\kappa\left(p_{2},-\varepsilon \xi_{\perp}^{2} /\left|\xi^{2}\right|\right.}=\frac{\kappa\left(p_{1},-v_{1}\right) \kappa\left(p_{2}, v_{2}\right)}{\kappa\left(p_{1}, v_{1}\right) \kappa\left(p_{2},-v_{2}\right)} .
$$

Then $\mathrm{Id}-Q$ in (4.9) is elliptic, and $P$ is a composition of an elliptic FIO of oder zero associated with $\mathcal{C}_{21}$ and an elliptic $\Psi D O$ (recall that all operators are microlocalized in small enough conic sets) of order 0 if $\sigma_{p}(Q)\left(p_{1}, \xi^{1}\right) \neq 1$.

Remark 5.1. We actually proved that $f=f_{1}+f_{2}$ can be recovered microlocally in $V^{1} \cup V^{2}$ by

$$
\begin{aligned}
& f_{1}=\left(X_{+, 2}^{-1} X_{+, 1}-X_{-, 2}^{-1} X_{-, 1}\right)^{-1}\left(X_{+, 2}^{-1} g_{+}-X_{-, 2}^{-1} g_{-}\right), \\
& f_{2}=\left(X_{+, 1}^{-1} X_{+, 2}-X_{-, 1}^{-1} X_{-, 2}\right)^{-1}\left(X_{+, 1}^{-1} g_{+}-X_{-, 1}^{-1} g_{-}\right),
\end{aligned}
$$


given $g_{ \pm}=X_{ \pm} f$. As above, all inverses are microlocal parametrices. In terms of $Q$, this could be written as

$$
f_{1}=(\mathrm{Id}-Q)^{-1} X_{+, 1}^{-1}\left(g_{+}-X_{+, 2} X_{-, 2}^{-1} g_{-}\right)
$$

with a similar formula for $f_{2}$ (which can be also reconstructed by solving the first or the second equation on (5.2) for $f_{2}$ ).

5.3. The attenuated X-ray transform. An important example satisfying (5.1) is the attenuated $\mathrm{X}$-ray transform. It as a weighted transform with weight

$$
\kappa(x, v)=e^{-\int_{0}^{\infty} \sigma\left(\gamma_{x, v}(s), \dot{\gamma}_{x, v}(s)\right) \mathrm{d} s},
$$

where $\sigma(x, v) \geq 0$ is the attenuation. The weight increases along the geodesic flow. More precisely, if $G$ is the generator of the geodesic flow, we have

$$
G \log \kappa=\sigma \geq 0 .
$$

If $\sigma>0$ along $\gamma_{x, v}$, then $\kappa$ is strictly increasing. Then (5.1) is trivially satisfied. In fact, for a fixed $\left(p_{1}, v_{1}, p_{2}, v_{2}\right),(5.1)$ is equivalent to requiring that $\sigma>0$ for at least one point on the geodesic through these points, between $p_{1}$ and $p_{2}$. We then get the following.

Theorem 5.2. Let $\left(M_{1}, g\right)$ and $M$ be as in Theorem 4.4. Assume that $C^{\infty} \ni \sigma>0$ in $M$ and let $X$ be the attenuated $X$-ray transform related to $\sigma$.

(a) If there are at most two conjugate points along each geodesic through $K$, then $X$ is stable, i.e., the conclusions of Theorem 4.4(a) hold.

(b) If there is a geodesic through $K$ with three (or more) conjugate points, then there is no stability, i.e., the conclusion of Theorem $4.4(b)$ holds.

Proof. Part (a) follows directly from (5.5) and a partition of unity on $S^{*} M$, as in the proof of Theorem 4.4.

If there are three or more points on $\gamma_{0}$ conjugate to each other, recovery of singularities is impossible. Indeed, let those points and the corresponding unit tangent vectors are $\left(p_{k}, v_{k}\right), k=$ $1, \ldots, N, N \geq 3$. We define the canonical relations $\mathcal{C}_{k l}=\mathcal{C}_{k}^{-1} \circ \mathcal{C}_{l}$ as before, and let $V^{k}$ be conic neighborhoods of the conormals at $p_{k}$. Then $\mathcal{C}_{k}\left(V^{k}\right)=\mathcal{V}$ for all $k$. Let $f_{k}$ have wave front sets in $V^{k}$. Given any $N-2$ of them, for example $f_{3}, \ldots f_{N}$, we can solve

$$
\begin{aligned}
& X_{+, 1} f_{1}+X_{+, 2} f_{2}+X_{+, 3} f_{3}+\ldots=g_{+}, \\
& X_{-, 1} f_{1}+X_{-, 2} f_{2}+X_{-, 3} f_{3}+\ldots=g_{-}
\end{aligned}
$$

for $f_{1}$ and $f_{2}$ microlocally. Therefore, we cannot recover the singularities and if, in particular, $X\left(f_{1}+\cdots+f_{N}\right) \in C^{\infty}(\mathcal{V})$, the last $N-2$ distributions can have arbitrary singularities on $V^{k}$, $k=3, \ldots, N$. This implies (b), see, e.g., [30].

\section{Numerical Experiments}

6.1. Cancellation of singularities. The first numerical experiment aims to illustrate Theorem 4.3. We choose $f_{1}$ and construct $f_{2}$ so that $f_{1}-f_{2}$ has fewer singularities than $f_{1}$.

We use the code developed in Matlab by the first author, whose detail may be found in [22]. The manifold $M$ is chosen to be the unit disk while the smaller neighborhood $U_{2}$ where the "artifacts" 
are expected, is the disk of center $(0,0.5)$ and radius 0.5 (both domains are displayed at the left of Fig. 3). We pick the (isotropic) metric from [22], taking the scalar expression

$$
g(x, y)=\exp \left(k \exp \left(-\frac{x^{2}+y^{2}}{2 \sigma^{2}}\right)\right), \quad \text { with } \quad \sigma=0.25, \quad k=1.2 .
$$

The manifold $(M, g)$ is not simple while the manifold $\left(U_{2}, g\right)$ is. We choose $f_{1}$ to be a Gaussian well concentrated near a single point, and we view this as an approximation of a delta function. The thick-marks in Figure 4, left, show the mesh chosen on the circle. The discretization of the initial directions is not visualized. The $\mathrm{X}$-ray transform $X f_{1}$ is supported on the ingoing boundary of $M$ and is parameterized in so-called "fan-beam" coordinates $(\beta, \alpha) \in[0,2 \pi] \times\left[-\frac{\pi}{2}, \frac{\pi}{2}\right]$, where $\beta$ locates the initial (boundary) point $\gamma(0)=\left(\begin{array}{c}\cos \beta \\ \sin \beta\end{array}\right)$ of the geodesic and $\alpha$ denotes the argument of its (unit) speed with respect to the inner normal, i.e. $\dot{\gamma}(0)=g^{-\frac{1}{2}}(\gamma(0))\left(\begin{array}{l}\cos (\beta+\pi+\alpha) \\ \sin (\beta+\pi+\alpha)\end{array}\right)$. The X-ray transform of a delta function is a delta function on a certain curve in $\mathcal{M}$. Its conjugate locus is above its center, with two folds connected in a cusp, see the second plot in Figure 5. The artifacts in the reconstruction of the delta should be supported above the conjugate locus and conormal to the fold part of it.
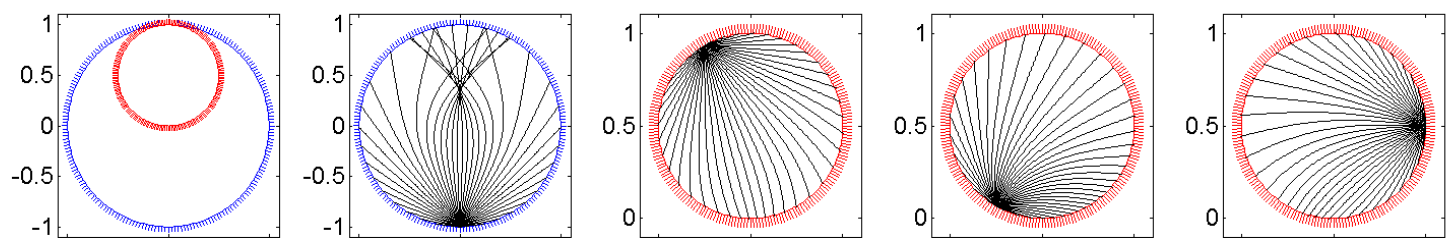

Figure 3. The computational domain and a few family of geodesics. The large disk is $M$ labeled as domain1, the smaller one is $U_{2}$, called domain2. $(M, g)$ has circular symmetry, so the geodesics from any other boundary point look similar on the second picture. $\left(U_{2}, g\right)$ has no symmetry, though it is simple (notice axes have changed on the three rightmost pictures, to fit the size of domain2).
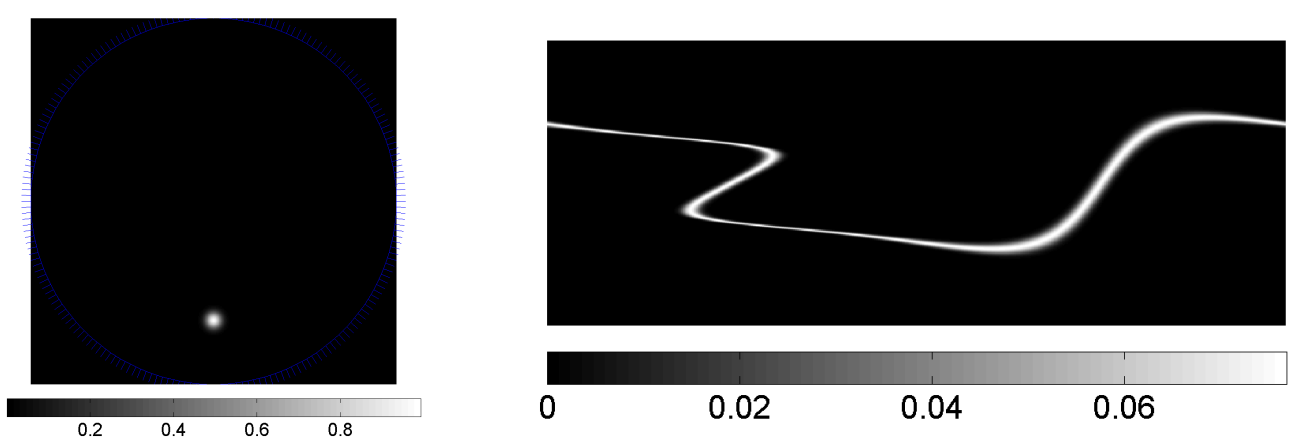

Figure 4. The function $f_{1}$ (left) and $X f_{1}$ (right). Observe on $X f_{1}$ that when the metric is non-simple, the X-ray transform of a delta function can no longer be expressed as a one-to-one $\alpha(\beta)$ function.

The goal next is to compute $F_{21} f_{1}=X_{2}^{-1} X_{1} f_{1}$ microlocally, i.e., to construct a function with a wave front set as that of $F_{21} f_{1}$ near the conjugate locus. We compute $X f_{1}$ first in $M$ and then 
we remap the data from $M$ to $U_{2}$ via free geodesic transport. The so remapped data does not fill the whole $\partial_{+} S U_{2}$ and may not belong to the range of $X$ on $U_{2}$ there. Still, a microlocal inversion is possible. On the remapped data, we apply the reconstruction formula on $U_{2}$ derived in [24] and implemented in [22], and call the reconstructed quantity $f_{2}$. This is equivalent, microlocally, to computing $X_{1} f$ first and and applying $X_{2}^{-1}$ to that, i.e., the result is $f_{2}=X_{2}^{-1} X_{1} f_{1}$ on some conic open set $V^{2}$ as above. Numerically, computing $X_{2}^{-1} X_{1} f_{1}$ is based on the following. We can reduce the problem $X_{2} f_{2}=X_{1} f_{1}$ (microlocally) to solving the Fredholm-type equation of the form

$$
f_{2}+W^{2} f_{2}=A X_{1} f_{1},
$$

where $W^{2}$ is an operator of order -1 when the metric is simple, whose Schwartz kernel is expressed in terms of the Jacobi fields $(a, b)$ as section 3.2 , but with Cauchy conditions on the boundary $(1,0)$ and $(0,1)$, respectively, and $A$ is an explicit approximate reconstruction formula. It is proved in [20] that $W^{2}$ is a contraction when the metric has curvature close enough to constant, though numerics in [22] indicate that considering $W^{2}$ a contraction and inverting the above equation via a Neumann series successfully reconstructs a function from its ray transform in all simple metrics considered. Once $f_{2}$ is constructed using this approach, we subtract it from $f_{1}$ (Figure 5 , left), then compute the forward data $X\left(f_{1}-f_{2}\right)$ on the large domain (see Fig. 6, left, where some singularities of $X f_{1}$ have been canceled). The function/distribution $f=f_{1}-f_{2}$, plotted in Figure 5 , is then the one with canceled singularities, by Theorem 4.3. Figure 6 illustrates the cancellations. Of course, only some open conic set of the singularities is canceled, corresponding to geodesics having conjugate points in $M$. In fact, it is clear from Figure 5 that the cancellation occurs near two directed vertical geodesics corresponding to a small strip around the horizontal medium in Figure 6.
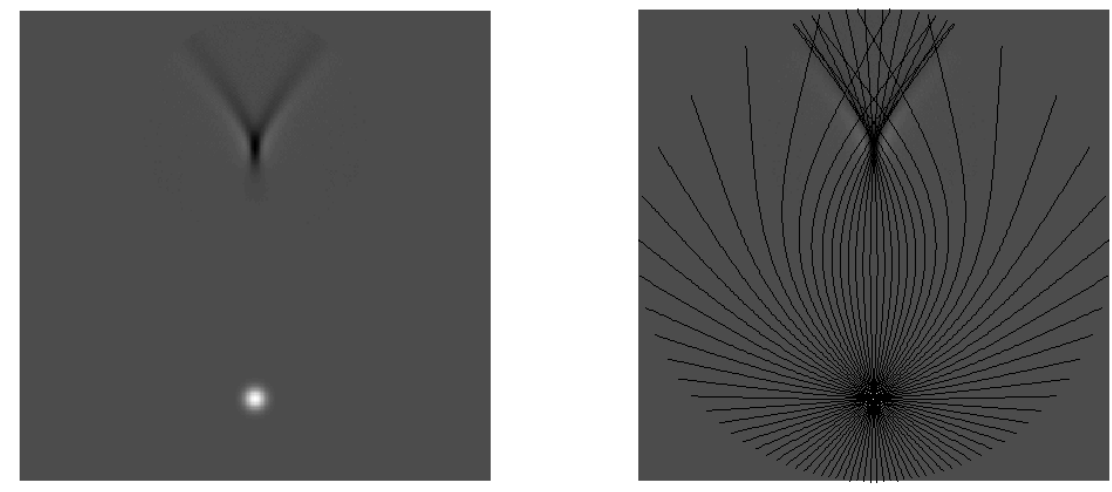

Figure 5. The function $f=f_{1}-f_{2}$ (left) and the same function with a few superimposed geodesics on it (right). The "artifact" $f_{2}$ appears as an approximate conormal distribution to the conjugate locus of the blob that $f_{1}$ represents. The gray scale has changed, and black now represents negative values, around -0.5 .

6.2. Artifacts in the reconstruction. We illustrate Theorem 4.5 now; what happens if we use $X^{*} X$ as an reconstruction attempt. If we apply this to the previous setup, we would get $f_{1}+f_{2}$ rather than $f_{1}-f_{2}$. Here we still consider the domain $M$ with the metric from (6.1) translated so that it is centered at $(0.2,0)$. Now, $f_{1}$ is a collection of peaked Gaussians alternating in signs (Fig. 7, left). Set $N=X^{*} X$. We apply $X^{*}$ to $X f$, and then $N$ again to get $N^{2} f$. The advantage to this is that locally near $p_{1}$ and near $p_{2}$, the parametrix is $-C \Delta_{g}$ instead of a square root of the 

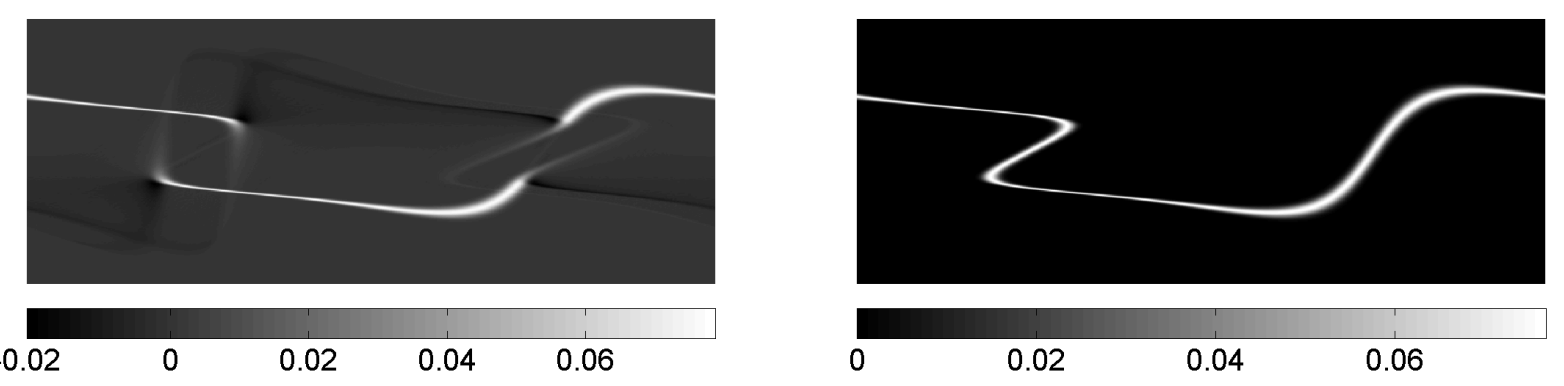

Figure 6. $X\left(f_{1}-f_{2}\right)$ (left) and $X f_{1}$ (right). Some singularities of $X f_{1}$ are nearly erased. The gray scale on the left is slightly different to allow for the negative values of $X\left(f_{1}-f_{2}\right)$.

latter. Then we apply $-C \Delta_{g}$ to get $-C \Delta_{g} N^{2} f$. Near $f_{1}$, this recovers $f_{1}$ up to an operator of order -1 applied to it. It also "recovers" the artifact $f_{2}$. The results are shown in Figure 7 .
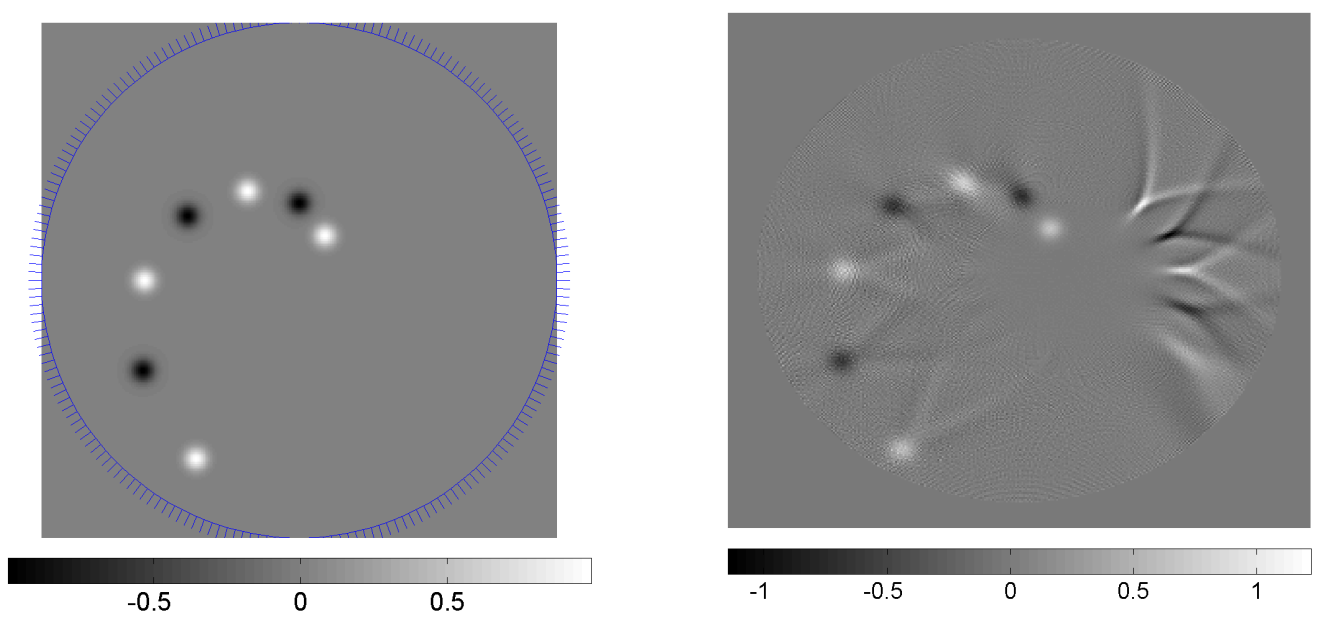

Figure 7. $f_{1}$ (left) and $-C \Delta_{g} N^{2} f_{1}$ (right).

The artifacts appear as an approximation of the union of the conjugate loci of each blob. Unlike the previous example, we see here $f_{1}+f_{2}$ (what we recover), not $f_{1}-f_{2}$ (what would cancel the singularities). The two blobs closer to the center create no artifacts because their conjugate loci are out of the disk $M$.

\section{REFERENCES}

[1] V. I. Arnol'd. Singularities of caustics and wave fronts, volume 62 of Mathematics and its Applications (Soviet Series). Kluwer Academic Publishers Group, Dordrecht, 1990.

[2] G. Bao and H. Zhang. Sensitivity analysis of an inverse problem for the wave equation with caustics. arXiv:1211.6220, 2012.

[3] M. I. Belishev and Y. V. Kurylev. To the reconstruction of a Riemannian manifold via its spectral data (BCmethod). Comm. Partial Differential Equations, 17(5-6):767-804, 1992. MR1177292. 
[4] M. Bellassoued and D. Dos Santos Ferreira. Stability estimates for the anisotropic wave equation from the Dirichlet-to-Neumann map. Inverse Probl. Imaging, 5(4):745-773, 2011.

[5] J. Boman and E. T. Quinto. Support theorems for real-analytic Radon transforms. Duke Math. J., 55(4):943-948, 1987.

[6] C. B. Croke. Rigidity and the distance between boundary points. J. Differential Geom., 33(2):445-464, 1991.

[7] C. B. Croke. Rigidity theorems in Riemannian geometry. In Geometric methods in inverse problems and PDE control, volume 137 of IMA Vol. Math. Appl., pages 47-72. Springer, New York, 2004.

[8] C. B. Croke, N. S. Dairbekov, and V. A. Sharafutdinov. Local boundary rigidity of a compact Riemannian manifold with curvature bounded above. Trans. Amer. Math. Soc., 352(9):3937-3956, 2000.

[9] J. J. Duistermaat and V. W. Guillemin. The spectrum of positive elliptic operators and periodic bicharacteristics. Invent. Math., 29(1):39-79, 1975.

[10] B. Frigyik, P. Stefanov, and G. Uhlmann. The X-ray transform for a generic family of curves and weights. J. Geom. Anal., 18(1):89-108, 2008.

[11] I. M. Gelfand, M. I. Graev, and Z. J. Shapiro. Differential forms and integral geometry. Funkcional. Anal. $i$ Priložen., 3(2):24-40, 1969.

[12] M. Golubitsky and V. Guillemin. Stable mappings and their singularities. Springer-Verlag, New York, 1973. Graduate Texts in Mathematics, Vol. 14.

[13] A. Greenleaf and G. Uhlmann. Nonlocal inversion formulas for the X-ray transform. Duke Math. J., 58(1):205$240,1989$.

[14] V. Guillemin. On some results of Gel'fand in integral geometry. In Pseudodifferential operators and applications (Notre Dame, Ind., 1984), volume 43 of Proc. Sympos. Pure Math., pages 149-155. Amer. Math. Soc., Providence, RI, 1985.

[15] V. Guillemin and S. Sternberg. Geometric asymptotics. American Mathematical Society, Providence, R.I., 1977. Mathematical Surveys, No. 14.

[16] S. Helgason. The Radon transform, volume 5 of Progress in Mathematics. Birkhäuser Boston Inc., Boston, MA, second edition, 1999.

[17] S. Holman. Microlocal analysis of the geodesic X-ray transform. private communication.

[18] L. Hörmander. The analysis of linear partial differential operators. III, volume 274. Springer-Verlag, Berlin, 1985. Pseudodifferential operators.

[19] L. Hörmander. The analysis of linear partial differential operators. IV, volume 275. Springer-Verlag, Berlin, 1985. Fourier integral operators.

[20] V. Krishnan. On the inversion formulas of Pestov and Uhlmann for the geodesic ray transform. J. Inv. Ill-Posed Problems, 18:401-408, 2010.

[21] V. P. Krishnan. A support theorem for the geodesic ray transform on functions. J. Fourier Anal. Appl., 15(4):515520, 2009.

[22] F. Monard. Numerical implementation of two-dimensional geodesic X-ray transforms and their inversion. to appear in SIAM J. Imaging Sciences, 2014. arXiv:1309.6042.

[23] C. Montalto. Stable determination of a simple metric, a covector field and a potential from the hyperbolic dirichlet-to-neumann map. Communications in Partial Differential Equations, 39(1):120-145, 2014.

[24] L. Pestov and G. Uhlmann. On characterization of the range and inversion formulas for the geodesic X-ray transform. Int. Math. Res. Not., (80):4331-4347, 2004.

[25] L. Pestov and G. Uhlmann. Two dimensional compact simple Riemannian manifolds are boundary distance rigid. Ann. of Math. (2), 161(2):1093-1110, 2005.

[26] E. T. Quinto. Radon transforms satisfying the Bolker assumption. In 75 years of Radon transform (Vienna, 1992), Conf. Proc. Lecture Notes Math. Phys., IV, pages 263-270. Int. Press, Cambridge, MA, 1994.

[27] V. Sharafutdinov. Variations of Dirichlet-to-Neumann map and deformation boundary rigidity of simple 2manifolds. J. Geom. Anal., 17(1):147-187, 2007.

[28] V. A. Sharafutdinov. Integral geometry of tensor fields. Inverse and Ill-posed Problems Series. VSP, Utrecht, 1994.

[29] P. Stefanov and G. Uhlmann. Rigidity for metrics with the same lengths of geodesics. Math. Res. Lett., 5(12):83-96, 1998.

[30] P. Stefanov and G. Uhlmann. Stability estimates for the hyperbolic Dirichlet to Neumann map in anisotropic media. J. Funct. Anal., 154(2):330-358, 1998.

[31] P. Stefanov and G. Uhlmann. Stability estimates for the X-ray transform of tensor fields and boundary rigidity. Duke Math. J., 123(3):445-467, 2004. 
[32] P. Stefanov and G. Uhlmann. Boundary rigidity and stability for generic simple metrics. J. Amer. Math. Soc., 18(4):975-1003, 2005.

[33] P. Stefanov and G. Uhlmann. Stable determination of generic simple metrics from the hyperbolic Dirichlet-toNeumann map. Int. Math. Res. Not., 17(17):1047-1061, 2005.

[34] P. Stefanov and G. Uhlmann. Boundary and lens rigidity, tensor tomography and analytic microlocal analysis. In Algebraic Analysis of Differential Equations. Springer, 2008.

[35] P. Stefanov and G. Uhlmann. Integral geometry of tensor fields on a class of non-simple Riemannian manifolds. Amer. J. Math., 130(1):239-268, 2008.

[36] P. Stefanov and G. Uhlmann. Local lens rigidity with incomplete data for a class of non-simple Riemannian manifolds. J. Differential Geom., 82(2):383-409, 2009.

[37] P. Stefanov and G. Uhlmann. The geodesic X-ray transform with fold caustics. Anal. PDE, 5-2:219-260, 2012.

[38] M. E. Taylor. Pseudodifferential operators, volume 34 of Princeton Mathematical Series. Princeton University Press, Princeton, N.J., 1981.

[39] G. Uhlmann and A. Vasy. The inverse problem for the local geodesic ray transform. preprint.

[40] F. W. Warner. The conjugate locus of a Riemannian manifold. Amer. J. Math., 87:575-604, 1965.

Department of Mathematics, University of Washington, Seattle, WA 98195

Department of Mathematics, Purdue University, West Lafayette, IN 47907

Department of Mathematics, University of Washington, Seattle, WA 98195 